\title{
Mental Health and Physical Health (Including HIV/AIDS)
}

A. Kagee, Stellenbosch University, Matieland, South Africa

M. Freeman, Human Sciences Research Council, Pretoria, South Africa

\section{Introduction}

According to the World Health Report 2001, over the past 20 years a fundamental and inseparable connection between mental and physical health has been convincingly established (WHO, 2001). Both mental and physical health are influenced by a combination of biological, psychological, and social factors. Thoughts, feelings, and behaviour have a major impact on physical health. Conversely, physical health has an important influence on mental health and well-being. The report notes two critical pathways through which this occurs: Physiological systems such as neuro-endocrine and immune functioning and health behaviour.

These pathways are not independent: Behaviour may affect physiology, while physiological functioning may in turn affect health behaviour. In this article, we examine the historical shift that has occurred from a dualistic conception of health and illness to the bio-psychosocial model that emphasizes an integration of mind and body. We explore this interrelationship using the examples of somatoform disorder, chronic pain, HIV/AIDS, cardiovascular disease, cancer, and diabetes. Finally, we focus on some of the somatic manifestations of mental illness.

\section{Historical Development of an Integrated Model: The Biomedical Model}

In the seventeenth century, the mind and body were considered to be two separate entities, largely unrelated to each other and influenced by discrete sets of factors. Rene' Descartes, a major proponent of a dualistic conceptualization of mind and body, considered it appropriate that the functioning of the body should fall in the realm of science, while the workings of the mind and soul should be the concern of philosophy and, subsequently psychology.

In the strongly religious atmosphere of this era, Cartesian dualism provided an important restraint on the influential Christian church from interfering in scientific activities that were considered previously taboo, such as the dissection of corpses, which was considered sinful and was forbidden by the European church. However, if it could be agreed that scientists would be concerned with the body and nothing else, it would no longer be the prerogative of the church to interfere in scientific affairs.

In return, the soul and mind would remain the domain of the clergy and outside of the realm of science. In this context, mind and body were separate and the boundary between the two was seen as impermeable. Dualism became highly influential in informing the biomedical model of health care. The medical model, whose hegemony went largely unchallenged for more than two centuries, embraces the view that illness is caused by internal or external agents that arrest or alter the normal functioning of the body. Such agents include bacteria, viruses, toxins, carcinogens, or genes.

In the medical model, the objective is to identify the etiological factors associated with poor health and rectify these so as to restore the body to optimal functioning. Accordingly, humans are chiefly biological organisms who can be understood by examining their constituent parts. In its extreme form, the only conceptual apparatuses required by the biomedical model to understand human functioning are physical and chemical in nature.

This emphasis has led to the ascendance of disciplines such as anatomy, physiology, and biochemistry in traditional biomedicine. Disease in the biomedical paradigm occurs when there is disruption in normal biological functioning, usually caused by an identifiable event of a physical or chemical nature. Medical treatment from this perspective involves physical or chemical agents to correct the disruption and restore the body to health.

\section{The Bio-psychosocial Model}

With further discoveries in the health sciences in the nineteenth and twentieth centuries, it became apparent that a dualistic conceptualization of health and illness was an inadequate explanatory model. It was instead recognized that the physiological regulation of the body's various systems (e.g., the digestive and respiratory systems) entailed a series of complex interactions and feedback loops involving a variety of variables. The utility of linear and mechanistic causal models of illness thus diminished and a greater emphasis was placed on a systemic understanding of human functioning. Thus, viruses and bacteria remained necessary conditions for the occurrence of illness but were no longer considered sufficient. Instead, disease is thought to occur as a result of the interaction between host systems and disease agents.

This interaction is characterized by complexity and nonlinearity, with an emphasis on systemic rather than mechanistic functioning. It has become accepted that human health and well-being are shaped by a multitude of factors that include behaviour, personality, cognitive style, and social, economic and political relationships. The bio-psychosocial model emphasizes the 
interaction of psychological, social, economic, behavioural, biological, and physical factors in influencing the functioning of human beings.

As such, it invokes a systemic understanding in which elements are arranged hierarchically so that change in one element of a system affects other parts. Human beings are highly complex systems that consist of organ systems, organs, tissue, cells, and chemical elements located in a historical, social, and economic context. Yet, while this context influences individuals, the environment is similarly affected by human interpretation, action, interaction, and social change. Thus, environment influences human behaviour and is also simultaneously influenced by it.

Similarly, behaviour may influence biological processes but may also be influenced by such processes. The bio-psychosocial model considers the boundary between the psychological and physical dimensions of human functioning to be a highly permeable mediator of reciprocal influences. The emphasis on multiple elements and their dynamic interplay provides a complex and nuanced understanding of the factors that influence health.

The bio-psychosocial model defines health and illness as 'the product of a combination of factors that include biological characteristics (e.g. genetic predisposition), behavioural factors (e.g. lifestyle, stress, health beliefs), and social conditions (e.g. cultural influences, family relationships, and social support)' (American Psychological Association, 2001).

Political and economic structures and systems as well as (their associated) health systems and approaches to financing of health care have also been found to directly affect health.

Mental and physical health intersects in various ways. While in reality it is often not possible to separate out these relationships, the following are important identifiable junctures between mental and physical health.

- Mental health status may impact on health risk behaviours;

- Mental problems may manifest as or impact on physical health problems;

- $\quad$ Diseases may attack both the brain and other organs or functions of the body;

- $\quad$ Physical ailments may affect mental functioning;

- A mental disorder may influence the course of physical disease;

- Medication given for the treatment of both mental and physical health problems may affect the other.

Having addressed some of the paradigmatic and theoretical considerations regarding mental and physical health, we now focus on specific conditions such as somatization, chronic pain, HIVand AIDS, cardiovascular disease, cancer, and diabetes. Our analysis is in keeping with the bio-psychosocial model to the extent that we de emphasize rigid divisions between the two dimensions of health.

\section{Health Conditions : Somatoform Disorders}

When physical symptoms occur in patients that are not fully explained by a general medical condition, these are usually referred to as somatoform disorders. In the category of somatoform disorders, the DSM IV TR includes somatization disorder (characterized by pain, gastrointestinal, and sexual problems), conversion disorder (involving unexplained symptoms that affect motor or sensory function), and hypochondriasis (characterized by the idea that one may have a serious disease, based on misinterpretation of bodily symptoms).

Complaints about pain, nausea, erectile dysfunction, paralysis, and excessive concerns about illness in the absence of objective evidence to support such concerns may fall into the category of somatization disorder. The features of this condition that differentiate it from a general medical condition are the involvement of more than one unrelated organ system, chronicity of complaints without the development of structural abnormalities, and the absence of laboratory evidence that suggests a medical condition.

Somatization has been shown to pose serious medical, social, and economic problems and may be difficult to manage clinically. Various theories have been postulated to explain somatoform disorders. We review the psychoanalytic and the communicative perspectives. The idea that physical symptoms could be removed by influencing the mind rose to prominence in the nineteenth century and formed the foundation of the work of psychologists such as Jean Charcot, Pierre Janet, Josef Breuer, and Sigmund Freud.

The psychoanalytic perspective advanced by Freud suggests that an unexplained physical symptom is a defence in response to anxiety from unacceptable unconscious conflict. The theory holds that the individual experiences anxiety because of an unacceptable idea or experience, against which the conversion is a defence. Psychic energy is then converted into a somatic symptom, which debilitates the physical organ. By this process, the somatic loss symbolizes the underlying psychic conflict. When physical symptoms occur in patients that are not fully explained by a general medical condition, these are usually referred to as somatoform disorders. 
In the category of somatoform disorders, the DSM IV TR includes somatization disorder (characterized by pain, gastrointestinal, and sexual problems), conversion disorder (involving unexplained symptoms that affect motor or sensory function), and hypochondriasis (characterized by the idea that one may have a serious disease, based on misinterpretation of bodily symptoms). Complaints about pain, nausea, erectile dysfunction, paralysis, and excessive concerns about illness in the absence of objective evidence to support such concerns may fall into the category of somatization disorder.

The features of this condition that differentiate it from a general medical condition are the involvement of more than one unrelated organ system, chronicity of complaints without the development of structural abnormalities, and the absence of laboratory evidence that suggests a medical condition.

Somatization has been shown to pose serious medical, social, and economic problems and may be difficult to manage clinically. Various theories have been postulated to explain somatoform disorders. We review the psychoanalytic and the communicative perspectives.

The idea that physical symptoms could be removed by influencing the mind rose to prominence in the nineteenth century and formed the foundation of the work of psychologists such as Jean Charcot, Pierre Janet, Josef Breuer, and Sigmund Freud. The psychoanalytic perspective advanced by Freud suggests that an unexplained physical symptom is a defence in response to anxiety from unacceptable unconscious conflict.

The theory holds that the individual experiences anxiety because of an unacceptable idea or experience, against which the conversion is a defence. Psychic energy is then converted into a somatic symptom, which debilitates the physical organ. By this process, the somatic loss symbolizes the underlying psychic conflict. The communicative perspective holds that somatising patients may be defending against a variety of feelings such as depression, guilt, or anger. The patient then uses the problem of somatization to cope with these emotions and negotiate personal interactions that may be considered stressful. In the communicative perspective, the condition of alexithymia has been associated with somatization.

This term has been used to refer to persons who have difficulty in expressing their feelings. Thus when asked to express feelings in response to loss, a typical response may be a report about physical symptoms such as headaches. There appears to be some evidence suggesting a relationship between somatization and alexithymia. Alexithymic persons have been shown to be particularly susceptible to somatoform disorders and psychosomatic problems.

Somatization has often been viewed as a defence against awareness of emotional distress or as a masked version of depression. In a population based epidemiologic survey, among respondents with five or more somatic symptoms, 63\% reported psychological problems and $50 \%$ met criteria for a psychiatric diagnosis. Among persons without somatic symptoms on the other hand, these figures were 7\% and 6\%, respectively (Simon and Von Korf, 1991).

Common diagnostic conditions associated with somatization include chronic fatigue syndrome (CFS) and fibromyalgia. CFS is considered a revision of a condition that first occurred in the nineteenth century named neurasthenia, which referred to undue exhaustion in the context of minimal physical effort. It has been demonstrated that CFS is often comorbid with psychiatric illness. Fibromyalgia is related to chronic fatigue and is characterized by muscle pain and tenderness. This condition is also often accompanied by depression and sleep disturbance.

In keeping with the bio-psychosocial paradigm, it is postulated that if the mind and brain transact, then, being regulated by the brain, organ systems are subject to influence by the mind and, in turn, anything that affects the mind (e.g., society and culture). As an example of the nonlinearity of the mind-body interaction, we examine the question of chronic pain and its association with psychological disturbance.

\section{Chronic Pain}

The traditional biomedical model of chronic pain is that it is caused by an identifiable disease state or tissue damage. Medical interventions are aimed at correcting the physical pathology with the intention of removing the experience of pain. While there have been considerable advances in fields such as anatomy and neurophysiology, much of patients' reported experiences of pain cannot be accounted for only by physical factors.

For example, there are many instances when patients have reported dissimilar subjective experiences of pain despite identical physical stimuli to produce them; the association between the extent of injury and the intensity of reported pain has been shown to be modest; it has been suggested that between $30 \%$ and $50 \%$ of patients seeking medical treatment may not have a specific diagnosable condition (Dworkin and Massoth, 1994); and the majority of patients who present with chronic back pain do not have a physical basis for their condition.

Many chronic pain researchers place a strong emphasis on the distinction between disease and illness. Disease refers to the objective biological event such as trauma or physiological changes to body tissue. Illness, on the other hand, refers to the patient's subjective appraisal of the biological event and is associated with the experience of physical discomfort, emotional distress, behavioural limitations, and psychosocial disruption. While the biomedical model has traditionally focused on disease, the emphasis of the bio-psychosocial model is primarily on illness. 
Chronic pain in terms of the bio-psychosocial model is best understood as the result of on-going and multifactorial processes in which there is a dynamic and reciprocal relationship between the biological, psychological, and social factors shaping patients' experiences. The dynamics of these reciprocal relationships may occur in at least three ways. First, biological factors initiate and maintain physical sensations, psychological factors may influence the manner in which a person appraises and perceives physiological signs and social factors may influence how patients respond behaviourally to these perceptions.

Second, psychological and mood related factors affect biological processes by having an effect on hormone production and the autonomic nervous system. Behavioural responses may also have an effect on biological factors, as in the case of a patient who refrains from strenuous activities in order to reduce symptoms of pain. Third, biological factors and pharmaceutical treatment can cause fatigue, influence a patient's ability to concentrate, and affect their ability to engage in certain activities. Various examples demonstrate the interrelationship between these factors.

For example, soldiers who sustain injuries on a battlefield report feeling considerably less pain than they would if the same injury had occurred elsewhere; athletes typically report feeling pain after a sports injury only once they are off the sports field; and patients who have the same degree of physical deterioration as measured by objective tests may have very different subjective perceptions of pain. Patients may also report pain in the absence of any objective evidence of tissue damage. These examples provide an indication of the complexity of the pain experience.

\section{Gate Control Theory}

Probably the most successful attempt at understanding perception of pain is gate control theory (Melzack and Wall, 1982). In his original work, Melzack noted that pain is an adaptive response to ensure the survival of the species as it ensures that a person avoids a stimulus in order to avoid injury, for example a hot plate that causes tissue damage. Gate control theory suggests that there are three systems that converge to affect the way in which pain is perceived, namely, sensory discriminative, motivational affective, and cognitive evaluative.

The model thus rejects the dichotomous view that pain is either physiological or psychological and suggests an integration of peripheral stimuli with psychological variables such as mood and anxiety in the experience of pain. The pain experience involves an on-going set of activities that is reflexive at the beginning and may be modified by excitatory and inhibitory influences and the level of activity in the CNS. GCT proposes that there is a series of neurophysiological mechanisms located in the dorsal horn of each segment of the spinal cord.

The activity of these mechanisms mediates the experience of pain and depends in part on both sensory information from the external environment (e.g., temperature and touch) and brain processes related to emotional state, past learning, and expectations. The meaning attributed to the stimulation is then transmitted back to the gate via nerve fibres that go from the brain to the spinal cord. In this way, the experience of pain is related to a combination of sensory input (tissue damage) and the psychological and behavioural state of the person.

\section{Chronic Pain and Psychopathology}

Emotional distress is common among persons experiencing chronic pain (Gatchel, 1996). Gatchel proposes a three stage model to account for the relationship between chronic pain and distress. In Stage 1, the individual experiences emotional distress as a consequence of experiencing pain when it is acute, as pain is most commonly associated with physical harm. In Stage 2 when the pain does not remit and becomes chronic, psychological reactions may include learned helplessness, depression, emotional distress, anger, and somatization.

While the model does not suppose a personality type that predisposes an individual to experiencing pain, it presumes that the nature and extent of these problems depend on the pre-existing psychological characteristics and social context of the person. There appears to be mixed evidence concerning whether depression is a consequence of chronic pain or whether chronic pain is part of a symptom cluster of depression. In Stage 3 of the model, as the behavioural or psychological problems persist, the person may adopt a sick role, which permits him or her to be excused from responsibilities and obligations.

Physical deconditioning may also occur alongside the progression of patients along the trajectory of this model, as a lack of physical activity may result in muscular atrophy and in turn in a decrease in physical capacity. Physical deconditioning may have a deleterious effect on emotional well-being and self-esteem, which may lead to additional psychological difficulties. Those chronic pain patients who experience depression may, as part of the symptom picture of this condition, experience a decrease in their level of motivation to engage in work, social, or recreational activities, which may in turn further contribute to physical deconditioning. 


\section{Chronic Pain and Depression}

There is considerable evidence of a close relationship between chronic pain and symptoms of depression. However, this relationship is complex, and possibly over lapping as the diagnostic criteria for mild depressive disorder also include some physical problems that could be attributed to chronic pain, such as sleep disturbance, energy loss, change in appetite, and weight gain or loss.

Criterion contamination of this nature makes the process of accurate diagnosis of depression difficult. The various efforts to determine the causal direction between pain and depression may be organized in the following manner (Dersh et al., 2002):

- $\quad$ the antecedent hypothesis, which holds that depression precedes the onset of chronic pain;

- $\quad$ the consequence hypothesis, which states that depression follows the onset of pain;

- the scar hypothesis, which speculates that prior episodes of depression predispose pain patients to further episodes of depression;

- the cognitive behavioural mediation hypothesis, which states that cognitions mediate the relationship between chronic pain and the development of depression;

- the common pathogenic mechanisms hypothesis, which states that both pain and depression have a common etiological factor.

In a review of several studies addressing these various hypotheses, little support was found for the antecedent hypothesis, but robust support was found for the consequence hypothesis and the cognitive behavioural mediation hypothesis, which are in many ways compatible with each other (Fishbain et al., 1997). Some support was also found for the scar and common mechanisms hypotheses.

The scar hypothesis assumes a genetic predisposition to depression and is supported by findings that suggest that a higher proportion of patients with chronic pain have family members with depression than those in the general population.

There is also evidence that common processes are involved in the mechanisms of pain and mood disturbance. For example, nociceptive (pain related) and affective pathways are thought to coincide anatomically; the neurotransmitters associated with mood disorders, namely norepinephrine and serotonin, have some involvement in the gate control mechanism described above; and antidepressant medication has been shown to relieve chronic pain (Dersh et al., 2002). It is apparent therefore that the relationship between chronic pain and depression is complex and dynamic, rather than linear and mechanistic.

\section{Chronic Pain and Other Mental Disorders}

Depression is by far the most common psychological association with chronic pain. Yet, it is evident that conditions such as substance abuse, anxiety, somatoform disorders, and personality disorders may be common among patients suffering chronic pain. The association between these disorders and chronic pain have received much less empirical scrutiny than mood disorders and the trajectory of their causal pathways awaits investigation.

\section{HIV/AIDS and Mental Health}

The complex interrelationship between mental and physical health is starkly exemplified with respect to HIV/AIDS. Five key mechanisms through which this occurs are presented.

\section{Mental Health Status as a Precursor to HIV/AIDS}

In the United States, people with severe mental illness are nearly 20 times more likely to be infected with HIV than the general population. Lack of appreciation of risk, impaired social interactions, low levels of assertiveness, low use of condoms, injecting drug use, multiple partners, and homelessness appear to be some of the reasons for the higher infection rates.

Higher rates of infection have not been reported in the few studies that have been conducted in developing countries, where the prevalence of HIV in people with mental illness has usually mirrored population prevalence. It is possible that because studies have primarily been done in closed systems of inpatient psychiatric institutions that people have been somewhat protected from the infection.

However, once infection does take root within a closed system, the chances of widespread infection are increased. Given high risk sexual behaviours in people with mental illness globally, higher rates in this grouping should be anticipated. 
Infiltration of HIV into the CNS is common, often resulting in HIV dementia and minor cognitive disorder. HIV can be detected in the cerebrospinal fluid of over $90 \%$ of asymptomatic patients while $75 \%$ of AIDS patients have been found to have brain pathology at autopsy. Between $30 \%$ and $50 \%$ of HIV seropositive individuals are estimated to experience some cognitive-motor problems (Grant et al., 1999).

HIV invades the brain early in the infection process and in a certain proportion of people psychotic symptoms manifest, especially in late stage AIDS. Manic episodes are above the population norm in people with HIV (around 5\%), especially at more advanced stages of the disease, and are the most common reason for psychiatric hospitalization in the HIV seropositive population in the United Kingdom.

\section{Mental Disorders and HIV/AIDS}

Studies of the mental health status of people infected with HIV have consistently found a higher prevalence of mental health problems than is found in community or clinical samples, ranging from relatively mild distress to a full mental disorder. Mood disorder is the most frequent psychiatric complication associated with people with HIV/AIDS. High levels of major depression, mild depressive disorder and dysthymia have been found in seropositive individuals. Bing et al. (2001) found a 36\% 1 year prevalence of depression among a large national sample of HIV positive men and women in the US.

In a meta-analysis of studies comparing HIV positive and HIV negative samples, Ciesla and Roberts (2001) showed that major depressive disorder occurred nearly twice as often among HIV positive than HIV negative patients. In a review of studies of mental health problems of HIV infected people in developing countries, Collins et al. (2006) also found a significantly higher prevalence of depressive symptoms among HIV positive people compared with controls. Feelings of anxiety and distress are a normal and arguably even a healthy response to a diagnosis of HIV. However anxiety may reach clinical levels and impair overall functioning and people’s capacity for adequate self-care.

The prevalence of anxiety disorders in studies in the US range from negligible to around 40\%. Anxiety can be provoked by the unpredictability of the virus and by certain milestones such as initial diagnosis, first opportunistic infection, declining CD4 count, or the onset or progression of an AIDS defining illness. Since the introduction of antiretroviral therapy (ART) in developed countries, the mental health and quality of life of people living with HIV/AIDS has improved consider ably. Firstly, the progressive neuropsychiatric progression of HIV is diminished.

Secondly people's psychological responses to living with HIV/AIDS are deeply affected by treatment. A number of cross sectional as well as longitudinal studies have shown decreased depression for people on ART. However, there is other evidence that suggests that ART does not itself alleviate depression and that a diagnosis of HIV remains profoundly distressing for most people.

In London, even though there was a significant decrease in the number of referrals for adjustment disorder and organic brain syndromes from pre ART to post ART eras, there was an increase in the proportion of people experiencing depressive disorders. People tended to have new problems and anxieties around forming relationships, disclosure, and demoralization around the side effects of medication.

\section{Mental Disorder May Influence Health Behaviours}

An HIV positive person needs to engage in a number of behaviours to maintain good health. For example, he or she must engage in protected sex to avoid reinfection, eat nutritious food, refrain from excessive use of alcohol, seek treatment for opportunistic infections when needed and, if antiretroviral treatment is required, adhere to the medication regimen (95\% adherence is needed). The person's psychological state is likely to affect his or her ability to engage in these behaviours. As part of the HIV/AIDS Treatment Adherence, Health Outcomes and Cost Study Group, Uldall et al. (2004) reviewed over 50 studies that examined mental illness and substance abuse and the impacts on adherence.

Mental health problems were found to be barriers to adherence in a number of community samples, for example in a population based cohort and in a national probability sample of people living with HIV/AIDS engaged in primary care. This association has been found to be particularly significant in women. While the introduction of HAART in the US has reduced HIV associated dementia, two studies have shown that mental flexibility was strongly associated with poor adherence. The effect of depression on HIV medication has also been extensively studied. At least eight studies have shown that adherence to antiretroviral medication is adversely affected by mood disturbance.

A number of studies have reported associations between adherence and generalized anxiety disorder, panic disorder, PTSD, recent trauma, and social phobia. Initial studies in Africa have pointed to high levels of ART adherence. However, in a recent analysis of African adherence research Gill et al. (2005) caution against complacency and conclude that adherence rates in Africa are in fact 
quite variable and often poor. They suggest that additional research is urgently needed to determine patient level barriers. They also suggest that ways of increasing adherence levels need to be found.

In some pilot treatment sites, people with mental health and substance abuse problems have been excluded from programs in order not to compromise the program. While it is not clear to what extent such exclusion corresponds with program success rates, given that mental health and substance abuse have been regularly found to be barriers to adherence in developed countries, this relationship seems possible and needs further exploration.

\section{Does Treatment of Mental Disorder Improve Adherence?}

The evidence that mental health problems impair HIV treatment is far more comprehensive than the evidence available on treating mental health problems and thereby improving ART adherence. Nonetheless, there is some research that indicates efficacy resulting from mental health and psychosocial interventions.

According to Uldall et al. (2004), most of the interventions that have been designed to improve adherence have focused on cognitive behavioural skills, such as helping individuals with remembering to take their medication and improving medication taking self efficacy, rather than treating mental health problems in order to improve adherence and overall health.

However, a retrospective study based on pharmaceutical records found that antiretroviral adherence was higher for depressed patients who received antidepressant medication than those who did not. It was also higher for those who adhered to their psychiatric medication. In a study on the impacts of self efficacy on HIV viral load and distress in women living with AIDS, Ironson et al. (2005) showed an improved course of illness linked to their SMART/EST intervention.

They suggest that this may be the effect of improved adherence linked to their intervention. One US study on severely nonadherent patients showed that continuous and personalized counselling improved adherence and virologic outcomes. Motivational interviewing has also been utilized to improve adherence, although with mixed results.

\section{$\underline{\text { Side Effects of Medication }}$}

In a minority of patients, mania and psychosis can occur due to the AIDS medication they receive such as AZT, 3TC, efavirenz, abacavir, and nevirapine. People taking efavirenz may experience nightmares and have other paranoid symptoms. These usually resolve within 2-3weeks but may persist. Patients who have had multiple episodes of depression are at particular risk of having negative reactions to efavirenz.

\section{Cardiovascular Disease}

The most common psychological attributes associated with persons with cardiac disease are type A behaviour pattern (TABP). TABP is a predisposition to think, feel, and act in a time urgent, aggressive, and impatient manner. This constellation of behaviours first reached the attention of researchers in the 1950s when two cardiologists, Friedman and Rosenman, observed that their heart patients behaved similarly to one another during clinic visits. Patients sat on the edge of their seats while waiting for appointments, as if they were ready to bolt at any instant. In fact, the waiting room seats became worn in a rather unusual way: only in the front.

The original work on TABP was conducted in the 1970s (Friedman and Rosenman, 1974), and it was consistently found that the combination of cognitive, emotional, and behavioural predispositions was predictive of coronary heart disease. These predispositions included time urgency, impatience, aggression toward others, and a propensity toward hostility. In accounting for the relationship between TABP and CHD, it is thought that appraising the world in a time urgent and hostile manner can lead to chronic psychosocial stress via a mechanism involving excessive sympathetic nervous system activation, which in turn leads to an exacerbation of coronary artery atherosclerosis.

While TABP is a well-documented antecedent of CHD, there have also been psychological effects of heart disease. Clinical depression is a prominent psychological feature of CHD patients and may be a strong predictor of death. In a large scale national community survey, $52.1 \%$ of heart patients displayed symptoms of depression, and of these $30.1 \%$ met the criteria for clinical depression (Purebl et al., 2006). However, other estimates have been somewhat lower. CHD patients who experience depression are considered to be at greater risk of dying of a subsequent heart attack than non-depressed patients.

Various pathways between depression and CAD have been considered, including behavioural mechanisms that involve poor adherence to medical and behavioural recommendations, diminished heart rate variability, stress induced ischemia, platelet activation, and immunological dysregulation (Faller, 2005). Studies of efficacy and effectiveness for depression have generally been conducted with non CHD patients and have provided support for psychological treatments such as cognitive behavioural 
therapy and interpersonal therapy. In addition, aerobic exercise as a means to elevate mood may have additional benefits of addressing the cardiovascular condition from which patients suffer.

\section{Cancer}

While many of the psychosocial concerns of cancer are unique to the specific type of cancer, there are several common psychological and behavioural factors that define most oncology patients. Nezu and colleagues (1999) have systematically identified the psychological issues that patients may face when confronting cancer. When first detecting symptoms, the patient may experience anxiety and fear, which in some cases may lead to a delay in seeking a diagnosis. The task at hand in this early stage of illness is to seek the most appropriate and available medical attention.

Upon diagnosis, many patients experience emotional distress, anxiety, anticipatory grief, and anger, have to engage with the stress of treatment decision making, and have to adjust to a new role and related responsibilities. The process of undergoing treatment may also in many cases present its own challenges. Treatment requires the marshalling of effort, energy, and support in combating cancer, accompanied by anxiety and grief with the loss of well-being. Common treatments for cancer include chemotherapy, radiation therapy, and surgery.

Chemotherapy is a systemic therapy that involves introducing medication into the body with the intention of destroying cancer cells. The side effects of chemotherapy occur because of the medication's inability to differentiate between cancerous cells and normal cells. Common side effects of chemotherapy include hair loss, mouth sores, diarrhea, nausea and emesis, loss of appetite, and fatigue. Radiation therapy is considered a local therapy in that it is directed at a specific area of the body that is affected by cancer. Similar to chemotherapy, radiation that destroys cancer cells may also harm non-cancerous ones; thus side effects are usually related to the area of the body being treated.

For example, radiation targeted at the abdomen may lead to diarrhea, while radiation directed at the mouth may lead to changes in taste and sensitivity. Surgery is a local treatment that usually involves the loss of body tissue that is affected by cancer, such as the breast, prostate, lung, or larynx.

Many patients may experience anxiety prior to surgery and some may experience emotional difficulties related to their altered body image. At post-treatment, many patients may be concerned with the fear of recurrence and anxiety as the health care team may be less available to provide support. Many patients who have been through treatment report re-valuating their life priorities.

Among patients who experience a recurrence of cancer, common emotional responses include disappointment, guilt, anxiety, anger, and grief. Treatment decision making is accompanied by psychological adaptation to treatment and follow up evaluation. When facing death from cancer that has metastasized to other parts of the body, common experiences among patients include fear of abandonment, suffering, and anxiety and sadness related to loss of control over their lives. Patients may engage in a reevaluation of their lives and try to find meaning to their experience with cancer.

In general, emotional distress is commonly experienced by many cancer patients, especially when their diagnosis is unexpected. Specifically, symptoms of depression and anxiety are common, while psychiatric disorders such as major depression and posttraumatic stress disorder occur in a minority of patients. It has been argued that cancer patients are no more likely to experience major depression than general medical patients. In a study of psychiatric morbidity of breast cancer patients, the prevalence estimates of emotional distress, major depression, and generalized anxiety disorder were 29\%, $9 \%$, and 6\%, respectively (Coyne et al., 2004).

Distress among cancer patients may be most appropriately ameliorated with social and family support, information about treatment options, and psychological counselling. For the minority of patients who meet the criteria for a psychiatric disorder, psychotropic medication is likely to be appropriate.

\section{Personality and Cancer}

The question of whether personality factors play a role in precipitating the onset of cancer is controversial. The major proponent of the view that certain personality characteristics are associated with cancer is Hans Eysenck, who has conducted several studies on the so called type C, or cancer prone personality (Eysenck, 2000). According to Eysenck, persons at increased risk of developing cancer tend to be unassertive, harmony seeking, unable to express emotions, and have difficulties in coping that may lead to helplessness and depression.

There appear to be some data to support this contention. For example, in a prospective study in which a sample of 1353 healthy persons were followed for 10 years until death, personality traits that involved blocking of feelings and needs in important relationships appeared to be associated with certain cancers, most notably breast cancer (Grossarth Maticek et al., 1997). Eysenck even went so far as to propose autonomy training to move personality characteristics away from type C tendencies (Eysenck, 2000). On the other hand, results of other investigations have yielded null findings on the relationship of personality factors and carcinogenesis. 
In a prospective study of 5133 adults, the incidence of cardiovascular disease was significantly predicted by emotional liability, behavioural control, and TABP, but the incidence of cancer was unrelated to these variables. In an examination of the relationship between personality factors and cancer, Amelang and Schmidt Rathiens (2003) conclude that personality factors are of little importance and explain less than $2 \%$ of the health-disease variance. These authors have shown that the significance of these variables has decreased over the past several years, while the biomedical predictors of carcinogenesis have remained robust. The conclusion of this review is that 'hypotheses addressing the causal relationships between personality and disease are of little value’ (Amelang and Schmidt Rathiens, 2003: 22).

It may well be the case that certain personality factors predispose certain people to engaging in behaviours that place them at greater risk for developing certain kinds of cancer. For example, the relationship between smoking and lung cancer has been well documented. However, the case for personality characteristics as a carcinogenic or even constituting a risk factor for cancer remains unconvincing.

\section{Diabetes}

Psychological difficulties common among endocrine patients include poor adherence to medication and lifestyle regimens, poor adjustment to the illness, an exacerbation of medical symptoms by stress, and psychiatric problems such as depression and anxiety. Aikens and Wagner (2003) classified 65 consecutive referrals seen in a behavioural endocrinology service in terms of the primary presenting problem or the corresponding DSM IV diagnosis: major depression (15\%), adjustment disorder (8\%), dysthymic disorder (8\%), specific phobia (8\%), regimen non-adherence (13\%), and stress affecting diabetes mellitus (21\%).

In most of the literature on the psychological and behavioural aspects of diabetes, treatment adherence is a salient theme. Medications that are prescribed following consultation with a medical professional are usually dispensed with an expectation of close to perfect adherence. Such expectations pertain to the dosage, timing, ingestion with specific foods, contraindications regarding ingestion with other medicines, and consistent adherence to the treatment regimen over time (World Health Organization, 2003). These details are of crucial importance in maximizing the health benefits from medical treatment.

Patient non-adherence may therefore have severe implications for the control of symptoms, recovery time, quality of life, and mortality. Among the factors associated with adherence in diabetes patients are health literacy, social support, and emotional problems. Health literacy implies an awareness of the importance of adherence even when actual symptoms are absent. Behavioural regimens such as restricted fat and sugar intake, exercise, and adherence to specific medications in many cases serve an important preventive function by controlling blood sugar and insulin levels.

In the absence of overt symptoms, for many patients adherence may appear to be unimportant. Yet, the longer term health consequences of non-adherence may be severe, as symptoms will inevitably develop. Considerable research has demonstrated consistently that social support is a strong predictor of medical adherence. Social support for adherence is defined as encouragement from family and friends for the patient to cooperate with the recommendations and prescriptions of a health professional.

The expression of concern and encouragement from others to engage in health promoting behaviours, including medication adherence, combine with social desirability needs on the part of the patient to yield higher rates of medical cooperation. An issue related to social support, namely the relationship between the health care worker and patient, has also been shown to be strongly associated with adherence. Data obtained by Roberts (2002) further suggest that medical providers viewed communicating with patients about adherence issues as an essential component of the health care service.

While the provider-patient relationship may ostensibly constitute an example of social support, it also extends beyond this. The health professional is often seen as a person of authority, in the possession of specific expertise that is unobtainable elsewhere, and as someone in whom the patient solely invests hope for assistance in the recovery process. Emotional problems such as depression appear to be common among patients with diabetes. Depressive symptoms such as loss of interest in activities, decreased energy, fatigue, difficulties in concentrating, remembering and making decisions, and appetite disturbance may seriously affect the extent to which patients are adherent to their dietary and lifestyle regimens.

Anxiety may be related to diabetes in at least three ways: Symptoms of anxiety may be produced by sympathetic nervous system responses to hyperglycemia; endocrine abnormalities may be exacerbated by normal physiological stress responses, and the psychological stress of living with a chronic illness and managing the challenging self-care tasks may have a general negative effect on mood. Hypoglycemic fear is also a concern for insulin dependent diabetes patients as hypoglycemia is a highly distressing experience that can cause serious physical consequences that include unconsciousness, coma, and in extreme cases, death. 
Somatic symptoms form part of the symptom picture of a number of psychiatric illnesses, most notably major depressive disorder. The symptoms of depression, for example, include weight loss or gain, sleep disturbance, and fatigue or loss of energy. When criterion contamination of this nature occurs, it may be suggested that physical symptoms are 'caused' by a mental condition. Such an appraisal may tend toward dualism and a distortion of a complex condition, which has both mental and physical features. Yet, from a treatment perspective it is essential to understand the etiological features of a disease.

In assessing patients for depression, the DSMIV TR criteria stipulate that these symptoms should not be due to the direct effects of a medical condition, such as hypothyroidism. Hypothyroidism, a condition in which the body lacks sufficient thyroid hormone and is caused by the inflammation of the thyroid gland, is said to mimic the symptoms of major depressive disorder. The symptoms of hypothyroidism thus similarly include fatigue, weakness, weight gain or difficulty losing weight, depressed mood, irritability, memory impairment, and a decreased libido, which can cause difficulties for mental health professionals in distinguishing between the two conditions.

There are clear implications for treatment if patients are misdiagnosed: Hypothyroidism is effectively treated with hormone therapy, while the most efficacious treatment for depression is a combination of antidepressant medication and psychological counselling. Among cancer patients as well, common somatic symptoms include pain, fatigue, weakness, and reduced energy. Symptoms such as these make the process of diagnosing depression and anxiety in cancer patients a complex process, again with important treatment implications. On the other hand, there are somatic symptoms that are associated with a mental illness as well.

In general, somatic symptoms of depression create diagnostic dilemmas in the assessment of patients with medical comorbidities because symptoms may be due to either the medical condition or depressive illness. In an effort to determine whether the etiology of somatic symptoms was due to an illness condition or mood disturbance among older patients, Drayer and colleagues (2005) showed that scores on measures of somatization, namely the Asberg Side Effects Rating Scale and the Utvalg for Kliniske Undersogelser (UKU), were significantly correlated with psychological symptoms of depression but not with medical comorbidities (Drayer et al., 2005).

These authors recommended that when assessing medical disorders with multiple somatic complaints, clinicians should consider the possibility that such symptoms may be due to depressive illness rather

than solely due to the medical disorder. Further, it may be necessary to assess the effects of antidepressant medication on somatic symptoms. For example, in a 32 week trial testing the effects of citalopram, a selective serotonin reuptake inhibitor, on somatic symptoms among patients with anxiety and anxious depression, it was concluded that somatic symptoms often improve with successful antidepressant medication management (Lenze et al., 2005).

\section{Health and Behaviour Change}

Health behaviour is a key determinant of overall health, and a person's health behaviour is in turn highly dependent on that person's mental health and psychological state. Many non-communicable diseases such as cardiovascular disease and diabetes are linked to unhealthy behaviour such as alcohol and tobacco use, poor diet, and a sedentary life style.

Health behaviour is also an important determinant of communicable diseases through, for example, unsafe sex practices. Health care seeking behaviour is similarly influenced by mental health. Three exemplars of theoretical models on health behaviour change need mention.

\section{Theory of Planned Behaviour}

The Theory of Planned Behaviour (TPB) postulates that the likelihood of an individual engaging in a health behaviour (for example, regular exercise) is correlated with the strength of his or her intention to engage in the behaviour. A behavioural intention represents an individual's commitment to act and is itself the outcome of a combination of several variables.

According to the TPB, the factors that directly influence intentions to engage in a health behaviour include the person's attitudes toward the behaviour, the person's perception of subjective group norms concerning the behaviour, and the extent to which the person perceives him or herself to have control concerning the behaviour (Fishbein, 2002).

\section{$\underline{\text { Health Belief Model }}$}

The Health Belief Model (HBM) hypothesizes that health related behaviour depends on the combination of several factors, namely, perceived susceptibility, perceived severity, perceived benefits, perceived barriers, cues to action, and self efficacy. Perceived susceptibility refers to an individual's opinion of the chances of contracting the illness condition. Perceived severity 
refers to an individual's opinion of how serious a condition and its consequences are. Perceived benefits refer to one's belief in the efficacy of the recommended health behaviour in reducing the risk or seriousness of the condition.

Perceived barriers refer to the perception of cost associated with adhering to a recommended health behaviour if it is likely to be beneficial in reducing or eliminating the perceived threat. Self efficacy refers to the level of confidence in one's ability to perform the health behaviour in question. Those persons who have low self efficacy will have low confidence in their ability, which will have an effect on the likelihood of the behaviour being performed. The HBM has been applied with considerable success to a range of health behaviours and populations, particularly preventive behaviours, such as diet, exercise, smoking cessation, vaccination, and contraception and sick role behaviours such as adherence to recommended medical treatments.

\section{The Transtheoretical Model}

This theory proposes that individuals progress through five interlocking stages in their effort to engage in health behaviours. Precontemplation is the time during which people are not seriously thinking about changing the behaviour that will permit the attainment of better health. In this stage, individuals are either unaware or underaware of their health problems and the need to alter their behaviour. During the stage of contemplation, people are aware that a health problem exists and have earnestly begun thinking about behaviour change but have not yet committed themselves to taking action.

The third stage of the model is preparation, in which the individual is preparing to enact the health behaviour in question. The next stage is the action stage, when individuals are making unambiguous changes in their behaviour, experiences, or environment in order to address health problems. The beginning of the maintenance stage is sometimes defined as 6 months following taking overt action to engage in the desired health behaviour. Progression through the stages defined by the model is not necessarily linear since in many instances relapses occur and individuals return to either the pre-contemplation or contemplation stages before finally succeeding in maintenance.

\section{Conclusion and Implications}

In defining health as a state of complete physical, mental, and social well-being and not merely the absence of disease and infirmity, the countries involved in developing this World Health Organization definition of health in 1948 portrayed great insight and understanding of the components of health. It is unlikely, however, that at the time they realized just how interdependent all the elements in the definition in fact are to health and illness. These relationships are now becoming clearer.

While much research is still needed to fully understand these relationships and mechanisms, conceptualizing the components as useful products of language that help explain different influences on health and well-being is an important step forward. Despite the evidence that the distinction between mental and physical health is permeable, in reality this distinction remains in the minds of many and in the health services of most countries. A number of shifts are needed to change this.

- Health worker education. The education of health workers rarely conceptualizes health as being a product of physical, mental, and social influences. Where this does happen, it is often in a linear manner. Even within many public health approaches where there is a growing emphasis on the social aspects of health, there is only minimal emphasis on the psychological or behavioural aspects. A comprehensive and integrated understanding of health needs to become the norm in order to change the way health workers think about and engage with their patients. Such an understanding should also result in health workers becoming more involved in prevention of health problems.

- Integration of mental and physical health care. The integration of mental health into general health care, where this has occurred, has been an important step forward in health care treatment. However, this approach is still in its infancy, especially in developing countries.

Separating physical and mental health services reinforces difference and mitigates against treating a whole person. Moreover, separation often leads to poorer accessibility of mental health services when, for example, only physical health care is provided at local clinics or hospitals.

- Stigma and discrimination. Mental health problems as well as some physical health problems, such as HIV/AIDS, are highly stigmatized in most countries and people are often discriminated against both by members of the public and health workers. Redressing stigma and discrimination is essential for good health within a population, as health seeking behaviour and health service provision may be adversely affected. Stigmatization also negatively influences the course of illness.

- $\quad$ Promoting health related behaviour. The relationships between lifestyle and treatment behaviours and health are being increasingly recognized as fundamental. Integrating more of the behavioural sciences with the health sciences is a crucial step that needs to be taken boldly. 
- $\quad$ Aikens JE and Wagner LI (2002) A review of empirically based psychological assessment and intervention procedures for adults with diabetes mellitus. Advances in Medical Psychotherapy and Psychodiagnosis 11: 5974.

- Amelang M and Schmidt Rathjens C (2003) Personality, cancer and coronary heart disease: Fictions and fact in the etiological research. Psychologische Rundschau 54(1): 1223.

- American Psychological Association (2001) Health Psychology, Mission Statement. http://www.health psych.org/mission.php (accessed October 2007).

- Bing EG, Burnam MA, Longshore D, et al. (2001) Psychiatric disorders and drug use among human immunodeficiency virus infected adults in the United States. Archives of General Psychiatry 58: 721728.

- $\quad$ Ciesla JA and Roberts JE (2001) Meta analysis of the relationship between HIV infection and risk for depressive disorders. American Journal of Psychiatry 158: 725730.

- Collins PY, Holman AR, Freeman M, and Patel V (2006) What is the relevance of mental health to HIV/AIDS care and treatment programs in developing countries? A systematic review. AIDS 20:1571 1582.

- Coyne JC, Palmer SC, Shapiro PJ, Thompson R, and De Michele A (2004) Distress, psychiatric morbidity, and prescriptions for psychotropic medication in a breast cancer waiting room sample. General Hospital Psychiatry 26 : 121 128.

- $\quad$ Dersh J, Polatin PB, and Gatchel RJ (2002) Chronic pain and psychopathology: Research findings and theoretical considerations. Psychosomatic Medicine 64(5): 773786.

- $\quad$ Drayer RA, Mulsant BH, Lenze EJ, et al. (2005) Somatic symptoms of depression in elderly patients with medical comorbidities. International Journal of Geriatric Psychiatry 20: 973982.

- $\quad$ Dworkin SF and Massoth DL (1994) Temporomandibular disorders and chronic pain: Disease or illness? Journal of Prosthetic Dentistry 72(1): 2938.

- Eysenck HJ (2000) Personality as a risk factor in cancer and coronary heart disease. In: Kenny DT, Carlson JG, McGuigan FJ, and Sheppard JL (eds.) Stress and Health: Research and Clinical Applications, pp. 291 318. Amsterdam the Netherlands: Harwood Academic Publishers.

- $\quad$ Faller H (2005) Depression. A prognostic factor in coronary artery disease/depression. Psychotherapeut 50(4): 265 273.

- $\quad$ Fishbain DA, Cutler RB, Rosomoff HL, and Rosomoff RS (1997) Chronic pain associated depression: Antecedent or consequence of chronic pain? A review. Clinical Journal of Pain 13(2): 116137.

- $\quad$ Fishbein M (2002) The role of theory in HIV prevention. In: Marks DF (ed.) The Health Psychology Reader, pp. 120 126. London: Sage.

- Gatchel RJ (1996) Psychological disorders and chronic pain: Cause and effect relationships. In: Gatchel RJ and Turk DC (eds.) Psychological Approaches to Pain Management: A Practitioner’s Handbook. New York: Guilford Press.

- $\quad$ Gill C, Hamer D, Simon J, et al. (2005) No room for complacency about adherence to antiretroviral therapy in Sub Saharan Africa. AIDS 12431249.

- $\quad$ Grant I, Marcotte TD, and Heaton RK (1999) Neurocognitive complications of HIV Disease. Psychological Science 10: 191195.

- Grossarth Maticek R, Eysenck HJ, Pfeifer A, Schmidt P, and Koppel G (1997) The specific action of different personality risk factors on cancer of the breast, cervix, corpus uteri and other types of cancer: A prospective investigation. Personality and Individual Differences 23(6): 949960.

- Ironson G, Weiss S, Lydston D, et al. (2005) The impact of improved self efficacy on HIV viral load and distress in culturally diverse women living with AIDS: The SMART/EST Women’s project. AIDS Care 17(2): 222236.

- $\quad$ Lenze EJ, Karp JF, Mulsant BH, et al. (2005) Somatic symptoms in late life anxiety: Treatment issues. Journal of Geriatric Psychiatry and Neurology 18(2): 8996.

- $\quad$ Melzack OR and Wall PD (1982) The Challenge of Pain. New York: Basic Books.

- Nezu AM, Nezu CM, Friedman SH, Faddis S, and Houts PS (1999) Helping Cancer Patients Cope. Washington, DC: American Psychological Association.

- $\quad$ Purebl G, Birkas E, Csoboth C, Szumska I, and Kopp MS (2006) The relationship of biological and psychological risk factors of cardiovascular disorder in a large scale national representative community survey. Behavioural Medicine 31(4):133 139.

- $\quad$ Roberts KJ (2002) Physician patient relationships, patient satisfaction, and antiretroviral medication adherence among HIV infected adults attending a public health clinic. AIDS Patient Care and STDS 16(1):43 50.

- Simon GE and VonKorff M (1991) Somatization and psychiatric disorder in the NIMH Epidemiologic Catchment Area study. American Journal of Psychiatry 148(11): 14941500.

- $\quad$ Uldall K, Palmer N, Whetten K, and Mellins C (2004) Adherence in people living with HIV/AIDS, mental illness, and chemical dependency: A review of the literature. AIDS Care 16(supplement 1):S71 S96.

- World Health Organization (2001) Mental Health: New Understanding, New Hope. Geneva, Switzerland: World Health Organization. World Health Organization (2003) Adherence to Long Term Therapies: Evidence for Action. Geneva, Switzerland: World Health Organization. 